\title{
Food insecurity and dietary intake by Supplemental Nutrition Assistance Program participation status among mainland US Puerto Rican adults after the 2009 American Recovery and Reinvestment Act
}

\author{
Amanda C McClain ${ }^{1}$, Katherine L Tucker ${ }^{2}$, Luis M Falcón ${ }^{3}$ and Josiemer Mattei ${ }^{1, *}$ (1) \\ 'Department of Nutrition, Harvard T.H. Chan School of Public Health, 655 Huntington Avenue, Boston, MA 02115 , \\ USA: ${ }^{2}$ Department of Biomedical and Nutritional Sciences, University of Massachusetts, Lowell, MA, USA: ${ }^{3}$ College of \\ Fine Arts, Humanities and Social Sciences, University of Massachusetts, Lowell, MA, USA
}

Submitted 2 October 2018: Final revision received 1 May 2019: Accepted 14 May 2019; First published online 9 August 2019

\begin{abstract}
Objective: The 2009 American Recovery and Reinvestment Act (ARRA) increased monthly Supplemental Nutrition Assistance Program (SNAP) benefits and expanded SNAP eligibility, yet limited evidence exists on the potential impact of ARRA on dietary intake among at-risk individuals. We aimed to examine pre-/post-ARRA differences in food insecurity (FI) and dietary intake by SNAP participation status.

Design: Pre/post analysis.

Setting: Boston, MA, USA.

Participants: Data were from the longitudinal Boston Puerto Rican Health Study (2007-2015). The US Department of Agriculture ten-item adult module assessed FI. A validated FFQ assessed dietary intake. Diet quality was assessed using the Alternate Healthy Eating Index-2010 (AHEI-2010). Self-reported pre-/post-ARRA household SNAP participation responses were categorized as: sustained ( $n$ 249), new ( $n$ 95) or discontinued ( $n$ 58). We estimated differences in odds of FI and in mean nutrient intakes and AHEI-2010 scores post-ARRA.

Results: Compared with pre-ARRA, OR (95\% CI) of FI post-ARRA were lower for all participants $(0.69(0.51,0.94))$, and within sustained $(0.63(0.43,0.92))$ but not within new $(0.94(0.49,1.80))$ or discontinued $(0.63(0.25,1.56))$ participants. Post-ARRA, total carbohydrate intake was higher, and alcohol intake was lower, for sustained and new participants, and dietary fibre was higher for sustained participants, compared with discontinued participants. Scores for AHEI-2010 and its components did not differ post-ARRA, except for lower alcohol intake for sustained $v$. discontinued participants.

Conclusions: Post-ARRA, FI decreased for sustained participants and some nutrient intakes were healthier for sustained and new participants. Continuing and expanding SNAP benefits and eligibility likely protects against FI and may improve dietary intake.
\end{abstract}

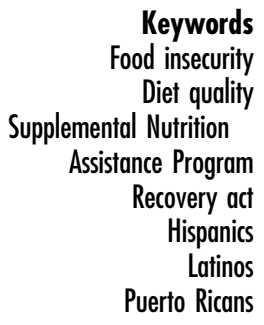

The 2007-2009 great recession in the USA was marked by a persistent rise in unemployment, declines in household income and inflation of food prices ${ }^{(1)}$. For low-income households, this translated into a larger proportion of income going towards food, further stretching household budgets $^{(1)}$. Temporary supplementary financial support for low-income households was provided by the American Recovery and Reinvestment Act of 2009
(ARRA), which both increased benefit levels for households participating in the federal Supplemental Nutrition Assistance Program (SNAP) and expanded SNAP eligibility for jobless adults without children ${ }^{(2)}$. One of ARRA's primary goals was to reduce household food insecurity (FI), defined as when consistent access to sufficient, healthy and safe food is limited or uncertain ${ }^{(3)}$. In December 2009, eight months after initiation of ARRA, 12.9\% of US 
residents received SNAP benefits compared with 10.6\% the previous year and the average monthly SNAP benefit amount had increased by $17 \%$. Food expenditures also increased and FI decreased ${ }^{(2)}$.

Increased access to SNAP benefits, as well as increased benefit amount, post-ARRA may have impacted food consumption patterns. Previous research has shown that SNAP participants had similar macronutrient and micronutrient intakes as income-eligible non-participants, but lower dietary quality ${ }^{(4)}$. Despite increased SNAP participation and improvement in food security post-ARRA, only one study has assessed dietary intake using cross-sectional data from the National Health and Nutrition Examination Survey (NHANES) $^{(5)}$ and two studies have assessed food security $^{(2,6)}$ pre- to post-ARRA initiation. Waehrer et al. found that while overall intakes of carbohydrates, protein, total fat, fibre, Na and vitamin $\mathrm{C}$ did not change post-ARRA SNAP expansion among households in the 2007-2010 NHANES $^{(5)}$, the overall proportion of energy from saturated fats increased significantly and mean diet quality scores decreased significantly ${ }^{(5)}$. Upon further investigation, ARRA SNAP expansion was associated with lower diet quality scores, particularly through higher intake of solid fats, alcohol and sugars, only among those with poor dietary intake pre-ARRA initiation and those with less education ${ }^{(5)}$. These nuanced findings for vulnerable populations and the overall limited evidence on ARRA-related diet changes underscore the major lack of evidence elucidating the role of ARRA or similar government-mandated SNAP expansions on dietary intake, especially among segments of the population most at risk for FI and poor diet and with the most potential to benefit. Furthermore, the crosssectional design of Waehrer et al.'s study ${ }^{(5)}$ limits accurate assessment of dietary outcomes.

We therefore aimed to evaluate pre- and post-ARRA SNAP expansion differences in FI and dietary intake among a longitudinal cohort of Puerto Rican adults living in the mainland USA, a population with a high prevalence of $\mathrm{FI}^{(7)}$ and SNAP participation ${ }^{(8)}$ as well as poor nutrient intake $^{(9)}$ and diet quality ${ }^{(10)}$ along with high rates of chronic disease ${ }^{(11,12)}$. Findings may help inform improved policy strategies that better equip Puerto Ricans and similar high-risk populations to lower FI and consume a healthy diet.

\section{Methods}

\section{Participants}

We analysed data from the Boston Puerto Rican Health Study (BPRHS), a longitudinal study on psychosocial stress, nutrition and health. Recruitment and data collection methods have been described in detail elsewhere ${ }^{(12)}$. Eligible participants were self-identified Puerto Rican adults (47 to 75 years) living in the greater Boston area, Massachusetts, and able to speak either English or
Spanish. Study staff recruited participants through doorto-door enumeration and community outreach strategies (e.g. festivals/fairs, radio spots). Between 2004 and 2015, participants completed study visits at three time points (baseline ( $n$ 1500), 2-year ( $n$ 1258) and 5-year ( $n$ 961)). Trained, bilingual interviewers obtained participants' written consent in participants' homes and administered questionnaires and performed anthropometric and blood pressure measurements in duplicate. Questionnaires collected information on demographics, psychological-based acculturation, FI, food assistance programme participation, health insurance coverage, depressive symptoms, dietary intake, physical activity, smoking behaviour and alcohol use. The study was approved by the Institutional Review Boards at Tufts Medical Center and at Northeastern University.

\section{Measures}

Food security status was assessed at baseline and 5-year follow-up using the valid and reliable US Department of Agriculture ten-item adult food security survey module, capturing the food security status of the respondent and other adult household members over the past 12 months ${ }^{(13)}$. Affirmative responses are summed and classified into one of four food security categories: high (0), marginal (1-2), low (3-5) or very low $(6-10)^{(14)}$. To maximize sample size, and in accordance with other studies on food security and diet ${ }^{(15)}$, we defined a binary food security status variable by combining high and marginal food security (food secure) and low and very low food security (food insecure). Participants self-reported SNAP participation at all three study visits.

Dietary intake was assessed using an adapted semiquantitative version of the National Cancer Institute Block-FFQ that incorporated Puerto Rican-appropriate foods and portion sizes. This adapted FFQ was previously validated in mainland US Puerto Ricans and more accurately represented intake than the original $\mathrm{FFQ}^{(16)}$. Participants were excluded if energy intakes were implausible $(<1674$ or $>20083 \mathrm{~kJ} / \mathrm{d}(<400$ or $>4800 \mathrm{kcal} / \mathrm{d}))$. Reported foods were used to compute intakes of total energy and nutrients. Nutrients were adjusted for energy intake using the residual method, in which the regression of nutrients $v$. total energy intake is performed to cancel out the correlated errors of energy intake and nutrients derived from the same $\mathrm{FFQ}^{(17)}$. We used the Alternate Healthy Eating Index-2010 (AHEI-2010) to assess diet quality. The AHEI-2010 consists of eleven dietary components associated with risk of chronic diseases, including vegetables (without potatoes), fruit (without fruit juice), whole grains, sugar-sweetened beverages and fruit juice, nuts and legumes, red and processed meat, trans-fatty acids, PUFA, $n-3$ fatty acids, $\mathrm{Na}$ and alcohol ${ }^{(18)}$. The minimum score for each component was 0 (worst) and the maximum score for each component was 10 (best), for a total 
AHEI-2010 score from 0 (lowest diet quality) to 110 (highest diet quality). Component scores were assigned based on the level to which the recommended amount of each dietary component was fulfilled.

\section{Covariates}

The potential confounders we considered were age, sex, income-to-poverty ratio, psychological-based acculturation, health insurance coverage, depressive symptoms, BMI, physical activity, smoking behaviour and alcohol intake. Income-to-poverty ratio was calculated by dividing total household income by the household's appropriate federal poverty threshold ${ }^{(19)}$. Psychological-based acculturation was assessed using an adapted ten-item Psychological Acculturation Scale that scored participants as being more Puerto Rican or more US American based on their subjective sense of belonging and emotional attachment to the two cultures. Scores ranged from 0 to 50 with higher scores indicating more US American identity ${ }^{(20)}$. Depressive symptoms were assessed using the twenty-item Center for Epidemiology Studies Depression (CESD) scale that captures the past-week frequency of experiencing feelings and behaviours associated with depression. Scores ranged from 0 to 60 with higher scores indicating greater depressive symptoms ${ }^{(21)}$. BMI was calculated from objective measures of weight and height $\left(\mathrm{kg} / \mathrm{m}^{2}\right)$. Physical activity was assessed using a modified Paffenbarger questionnaire and a score created that summed the total hours spent in typical activities over a $24 \mathrm{~h}$ period, multiplied by weighting factors associated with activity intensity ${ }^{(22)}$. Self-reported history and frequency of smoking and alcohol intake were collected and defined as 'never', 'past, but not current' and 'current' smoker or consumption of alcohol.

\section{Statistical analyses}

Because we were interested in comparing pre- and postARRA SNAP expansion values, we restricted our analytical sample ( $n$ 402) to participants with at least one of the three study visits pre-ARRA SNAP expansion initiation (1 April 2009) and one study visit post-ARRA SNAP expansion initiation but before the policy ended (1 November 2013). For all participants in the analytical sample, these two visits were the 2-year (pre-ARRA) and 5-year (post-ARRA) follow-up visits. Thus, we used SNAP participation and dietary intake at 2-year and 5-year follow-up. Food security status was used at baseline and 5-year follow-up, as it was not collected at 2-year follow-up. We categorized participants based on their SNAP participation status pre- and post-ARRA initiation: (i) sustained participants (SNAP participation both pre- and post-ARRA initiation); (ii) new participants (SNAP participation only post-ARRA initiation); and (iii) discontinued participants (SNAP participation only pre-ARRA initiation). Ineligible non-participants between 150 and $250 \%$ of the federal poverty line have previously been defined as a comparison group in similar analyses, because they are almost SNAP-eligible and are classified as low-income for many public health insurance programmes ${ }^{(5)}$. However, the sample size for ineligible nonparticipants in the BPRHS was too small ( $n$ 23) so only SNAP participants were considered in these analyses.

Descriptive analyses included paired $t$ tests to compare values pre- and post-ARRA for continuous variables, ANOVA to compare continuous variables by SNAP participation status, and $\chi^{2}$ tests (or Fisher exact test when appropriate) to compare categorical variables pre- and post-ARRA and by SNAP participation status. We estimated differences in odds of FI post-ARRA SNAP expansion, compared with pre-ARRA, using generalized estimating equations adjusted for sex, educational attainment, and pre-ARRA age, income-to-poverty ratio, psychological acculturation, health insurance and depressive symptoms. We ran this model first with our entire analytical sample, and then individual models for each SNAP participation status category. We used repeated-measures mixed models to estimate mean nutrient intakes and AHEI-2010 total and component scores post-ARRA by SNAP participation status, adjusting for the same covariates as above in addition to pre-ARRA nutrient values or AHEI-2010 total and component scores and BMI. We employed the Tukey-Kramer method to adjust for multiple pairwise comparisons. We used repeated-measures mixed models instead of difference-in-difference models because our sample sizes for new and discontinued participants were small. Mixed models retain observations with missing covariate data, unlike difference-in-difference models. Analyses were conducted in the statistical software package SAS version 9.4 with significance levels set at $P<0.05$.

\section{Results}

\section{Participant characteristics}

Of the 402 SNAP participants from pre- to post-ARRA SNAP expansion, 249 were sustained, ninety-five were new and fifty-eight were discontinued participants, indicating a $9 \%$ increase in SNAP participation. Participant characteristics pre- and post-ARRA SNAP expansion by SNAP participation status are reported in Table 1. Among sustained SNAP participants post-ARRA, compared with pre-ARRA, a higher proportion had children in the household and reported being food secure, and a lower proportion reported being married or partnered, current smokers or current drinkers. Mean household size, psychological acculturation and BMI were also lower post-ARRA among sustained participants. Similar significant trends were documented among new and discontinued SNAP participants from pre- to post-ARRA, except for mean household size for new and discontinued participants, and psychological acculturation and food security among new participants. New and discontinued participants also had a lower 
Table 1 Sociodemographic, sociocultural and health characteristics from pre-ARRA to post-ARRA SNAP expansion by SNAP participation status in the Boston Puerto Rican Health Study ( $n$ 402)†

\begin{tabular}{|c|c|c|c|c|c|c|c|c|c|c|c|c|}
\hline \multirow[b]{2}{*}{ Characteristic } & \multicolumn{4}{|c|}{ Sustained (n 249) } & \multicolumn{4}{|c|}{ New $(n 95)$} & \multicolumn{4}{|c|}{ Discontinued ( $n$ 58) } \\
\hline & $\begin{array}{l}\text { Pre } \\
\text { (mean } \\
\text { or \%) }\end{array}$ & SD & $\begin{array}{l}\text { Post } \\
\text { (mean } \\
\text { or \%) }\end{array}$ & SD & $\begin{array}{l}\text { Pre } \\
\text { (mean } \\
\text { or \%) }\end{array}$ & SD & $\begin{array}{l}\text { Post } \\
\text { (mean } \\
\text { or \%) }\end{array}$ & SD & $\begin{array}{l}\text { Pre } \\
\text { (mean } \\
\text { or \%) }\end{array}$ & SD & $\begin{array}{l}\text { Post } \\
\text { (mean } \\
\text { or \%) }\end{array}$ & SD \\
\hline Age (years), mean and SD & $60 \cdot 0$ & $7 \cdot 3$ & $64 \cdot 3^{* * \star *}$ & $7 \cdot 3$ & $60 \cdot 2$ & 7.5 & $64 \cdot 7^{\star \star \star \star}$ & 7.5 & $58 \cdot 7$ & $7 \cdot 3$ & $63 \cdot 0^{\star \star \star \star}$ & $7 \cdot 4$ \\
\hline Female $(\%)$ & 79.5 & - & - & - & $76 \cdot \overline{8}$ & - & - & - & $77 \cdot 6$ & - & - & - \\
\hline $\begin{array}{l}\text { Total household income } \\
\text { (\$US), mean and SD }\end{array}$ & 12746 & 7174 & 13345 & 5940 & 14831 & 9222 & 16087 & 13104 & 17628 & 23736 & 17164 & 12722 \\
\hline $\begin{array}{l}\text { Income-to-poverty ratio, } \\
\text { mean and SD }\end{array}$ & $93 \cdot 3$ & $38 \cdot 3$ & $97 \cdot 9$ & $35 \cdot 8$ & 102 & 53 & 101 & 59 & 116 & 116 & 116 & 88 \\
\hline $\begin{array}{l}\leq 8 \text { th grade educational } \\
\text { attainment }(\%)\end{array}$ & 61.9 & - & - & - & $45 \cdot 3$ & - & - & - & $57 \cdot 9$ & - & - & - \\
\hline Currently working (\%) & $3 \cdot 6$ & - & 1.6 & - & $17 \cdot 9$ & - & $7 \cdot 4^{*}$ & - & $15 \cdot 5$ & - & $12 \cdot 1^{\star \star \star}$ & - \\
\hline \multicolumn{13}{|l|}{ Marital status (\%) } \\
\hline Married/partnered & $25 \cdot 0$ & - & $21 \cdot 4^{\star \star \star \star}$ & - & 29.5 & - & $22 \cdot 1^{\star \star \star \star}$ & - & $32 \cdot 8$ & - & $29 \cdot 3^{\star \star \star \star}$ & - \\
\hline $\begin{array}{l}\text { Divorced/separated/ } \\
\text { widowed }\end{array}$ & $60 \cdot 5$ & - & $57 \cdot 7$ & - & $51 \cdot 6$ & - & $56 \cdot 8$ & - & 53.5 & - & $55 \cdot 2$ & - \\
\hline Never married & 14.5 & - & $21 \cdot 0$ & - & $19 \cdot 0$ & - & $21 \cdot 1$ & - & $13 \cdot 8$ & - & $15 \cdot 5$ & - \\
\hline $\begin{array}{l}\text { Household size, mean } \\
\text { and SD }\end{array}$ & $2 \cdot 0$ & 1.4 & $1 \cdot 7^{\star \star \star}$ & $1 \cdot 1$ & $2 \cdot 4$ & 1.6 & $2 \cdot 2$ & 1.6 & $2 \cdot 2$ & $1 \cdot 1$ & $2 \cdot 0$ & 1.3 \\
\hline $\begin{array}{l}\text { Children }<13 \text { years old } \\
\text { present in household (\%) }\end{array}$ & $12 \cdot 5$ & - & $8 \cdot 0^{* * * *}$ & - & $20 \cdot 0$ & - & $14 \cdot 7^{\star}$ & - & $15 \cdot 5$ & - & $12 \cdot 1^{\star \star \star}$ & - \\
\hline $\begin{array}{l}\text { Psychological acculturation } \\
\text { score } \neq \text {, mean and SD }\end{array}$ & $16 \cdot 6$ & $6 \cdot 1$ & $14 \cdot 7^{\star \star \star}$ & $7 \cdot 0$ & $17 \cdot 8$ & 6.5 & $16 \cdot 4$ & $7 \cdot 1$ & $17 \cdot 0$ & $5 \cdot 9$ & $14 \cdot 1^{\star *}$ & $6 \cdot 2$ \\
\hline $\begin{array}{l}\text { Depressive symptoms§, } \\
\text { mean and SD }\end{array}$ & $19 \cdot 9$ & $13 \cdot 0$ & $20 \cdot 4$ & $10 \cdot 5$ & $19 \cdot 8$ & $13 \cdot 2$ & $18 \cdot 6$ & $9 \cdot 5$ & $18 \cdot 0$ & $11 \cdot 6$ & $20 \cdot 7$ & $9 \cdot 1$ \\
\hline $\begin{array}{l}\text { Currently have health } \\
\text { insurance (\%) }\end{array}$ & $81 \cdot 5$ & - & $96 \cdot 8$ & - & $74 \cdot 2$ & - & $95 \cdot 7$ & - & 84.5 & - & $96 \cdot 6$ & - \\
\hline \multicolumn{13}{|l|}{ Smoking status (\%) } \\
\hline Never & $45 \cdot 6$ & - & $45 \cdot 6^{\star \star \star \star}$ & - & $46 \cdot 7$ & - & $46 \cdot 7^{\star \star \star \star}$ & - & $53 \cdot 6$ & - & $53 \cdot 6^{\star \star \star \star}$ & - \\
\hline Past, but not current & $28 \cdot 6$ & - & 34.9 & - & $37 \cdot 0$ & - & $37 \cdot 0$ & - & $25 \cdot 0$ & - & $30 \cdot 4$ & - \\
\hline Current & $25 \cdot 7$ & - & 19.5 & - & $16 \cdot 3$ & - & $16 \cdot 3$ & - & 21.4 & - & $16 \cdot 1$ & - \\
\hline \multicolumn{13}{|l|}{ Alcohol intake status (\%) } \\
\hline Never & $32 \cdot 4$ & - & $31 \cdot 6^{\star * \star *}$ & - & $28 \cdot 7$ & - & $26 \cdot 6^{\star * \star *}$ & - & $28 \cdot 1$ & - & $28 \cdot 1^{\star * \star *}$ & - \\
\hline Past, but not current & $36 \cdot 0$ & - & $48 \cdot 6$ & - & 40.4 & - & 48.9 & - & 38.6 & - & $49 \cdot 1$ & - \\
\hline Current & $31 \cdot 6$ & - & $19 \cdot 8$ & - & $30 \cdot 9$ & - & 24.5 & - & $33 \cdot 3$ & - & $22 \cdot 8$ & - \\
\hline $\begin{array}{l}\text { Physical activity score } \| \text {, } \\
\text { mean and SD }\end{array}$ & $30 \cdot 6$ & $3 \cdot 9$ & $30 \cdot 7$ & $5 \cdot 6$ & $31 \cdot 1$ & 3.4 & $32 \cdot 2$ & $6 \cdot 4$ & $30 \cdot 3$ & $3 \cdot 7$ & $32 \cdot 2^{*}$ & $6 \cdot 5$ \\
\hline BMI $\left(\mathrm{kg} / \mathrm{m}^{2}\right)$, mean and SD & $32 \cdot 5$ & $7 \cdot 0$ & $31 \cdot 1^{\star * * *}$ & $6 \cdot 5$ & $32 \cdot 8$ & $6 \cdot 5$ & $31.9^{*}$ & $7 \cdot 1$ & $31 \cdot 7$ & $6 \cdot 0$ & $30 \cdot 2^{\star * \star *}$ & $19 \cdot 7$ \\
\hline $\begin{array}{l}\mathrm{AHEI}-2010 \text { score } \| \text {, mean } \\
\text { and SD }\end{array}$ & $51 \cdot 9$ & $6 \cdot 8$ & $52 \cdot 2$ & $7 \cdot 7$ & $53 \cdot 2$ & $7 \cdot 4$ & $51 \cdot 2$ & $8 \cdot 3$ & $51 \cdot 6$ & $6 \cdot 9$ & $50 \cdot 1$ & $9 \cdot 3$ \\
\hline \multicolumn{13}{|l|}{ Food security†† (\%) } \\
\hline Food secure & 71.1 & - & $78 \cdot 7^{\star \star \star \star}$ & - & 77.9 & - & 77.9 & - & 75.4 & - & $82 \cdot 5^{\star \star}$ & - \\
\hline Low food security & $19 \cdot 7$ & - & 14.9 & - & 14.7 & - & $16 \cdot 8$ & - & $17 \cdot 5$ & - & $15 \cdot 8$ & - \\
\hline Very low food security & $9 \cdot 2$ & - & $6 \cdot 4$ & - & $7 \cdot 4$ & - & $5 \cdot 3$ & - & $7 \cdot 0$ & - & 1.8 & - \\
\hline
\end{tabular}

ARRA, 2009 American Recovery and Reinvestment Act; SNAP, Supplemental Nutrition Assistance Program; AHEI-2010, Alternate Healthy Eating Index-2010.

${ }^{\star} P<0.05,{ }^{\star \star} P<0.01,{ }^{\star \star \star} P<0.001,{ }^{\star \star \star \star} P<0.0001$ for trend.

†Analyses included paired $t$ tests for continuous variables pre-/post-ARRA (mean and SD) and $\chi^{2}$ (or Fisher exact test when appropriate) for categorical variables (\%).

$\ddagger$ Psychological acculturation score assessed degree of subjective sense of belonging and emotional attachment to US and Puerto Rican cultures. Scores ranged from 0 to 50 with higher scores indicating more US American identity.

§Depressive symptoms over the past week were assessed by the Center for Epidemiology Studies - Depression (CESD) scale with, higher scores indicating greater depressive symptoms (scores ranged from 0 to 60 ).

॥Physical activity score captured total time spent in light, moderate and vigorous activity, with higher scores indicating greater physical activity.

IThe AHEI-2010 assessed dietary quality using eleven dietary components. Higher scores indicate higher diet quality (scores range from 0 to 110 ).

t†Food security status was assessed using the US Department of Agriculture ten-item adult food security module. Participants with full or marginal food security were categorized as food secure. Participants with low or very low food security were categorized as food insecure.

\section{Food insecurity after the 2009 American Recovery and Reinvestment Act expansion of the Supplemental Nutrition Assistance Program}

Among the entire sample of SNAP participants, odds of FI post-ARRA were significantly lower compared with preARRA (OR (95\% CI): 0.69 (0.51, 0.94); Fig. 1). Within sustained participants, odds of FI were also lower post-ARRA

material, Supplemental Table S1). 


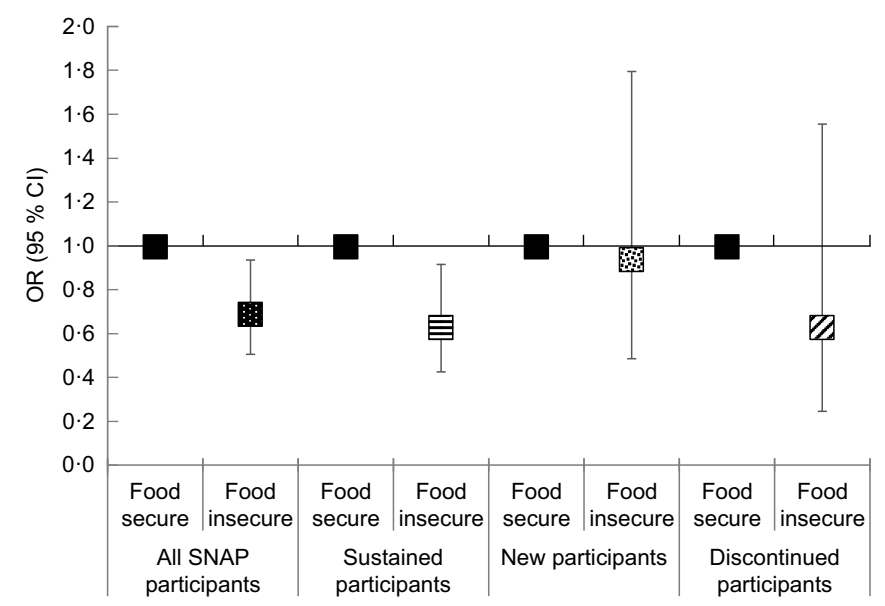

Fig. 1 OR, with $95 \% \mathrm{Cl}$ represented by vertical bars, of being food insecure post-ARRA SNAP expansion for the total sample (目) and within each SNAP participation status (트, sustained; 铗, new; $\mathbb{2}$, discontinued), adjusted for pre-ARRA sociodemographic, sociocultural and health factors, in the Boston Puerto Rican Health Study ( $n$ 402) (ARRA, 2009 American Recovery and Reinvestment Act; SNAP, Supplemental Nutrition Assistance Program)

compared with pre-ARRA $(0.63(0.43,0.92))$. Odds of FI post-ARRA, compared with pre-ARRA, were not significantly different within new $(0.94(0.49,1.80))$ or discontinued $(0.63(0.25,1.56))$ participants.

\section{Nutrient intakes after the 2009 American Recovery and Reinvestment Act expansion of the Supplemental Nutrition Assistance Program} Mean nutrient intakes post-ARRA SNAP expansion by SNAP participation status are reported in Table 2. PostARRA intakes of total energy, fat or protein, or of any type of fat or protein, or of key micronutrients did not differ by SNAP participation status. Similarly, post-ARRA AHEI-2010 scores and most component scores did not differ by SNAP participation status. Total carbohydrate intake was higher post-ARRA for sustained (mean (SE): 215 (1.9) g/d) and new $(216(2.6) \mathrm{g} / \mathrm{d})$ participants compared with discontinued participants $(204(3.4) \mathrm{g} / \mathrm{d})$, and alcohol intake was lower post-ARRA for sustained $(4.5(0.8) \mathrm{g} / \mathrm{d})$ and new $(5 \cdot 1(1 \cdot 1) \mathrm{g} / \mathrm{d})$ participants compared with discontinued participants $(9.7(1.5) \mathrm{g} / \mathrm{d})$. Similarly, sustained participants had lower AHEI-2010 alcohol component score (3.6 (0.1)) compared with discontinued participants (2.9 $(0 \cdot 2))$. Dietary fibre intake was also higher post-ARRA for sustained participants $(19.0(0.4) \mathrm{g} / \mathrm{d})$ compared with discontinued participants $(17.5(0.6) \mathrm{g} / \mathrm{d})$. No other nutrient differed by SNAP participant status post-ARRA.

\section{Discussion}

The findings from the present study demonstrate that Puerto Rican adults participating in SNAP, primarily sustained participants, experienced lower odds of FI postARRA SNAP expansion, suggesting that SNAP continuity and expanded benefits were influential. The post-ARRA
SNAP expansion period also yielded higher mean total carbohydrate intake for sustained and new participants and higher mean dietary fibre intake for sustained participants, compared with discontinued participants. Notably, mean alcohol intake was lower post-ARRA SNAP expansion for sustained and new participants, compared with discontinued participants.

SNAP has previously shown success in improving food security among low-income households, the primary objective of the programme ${ }^{(23)}$. Our findings contribute additional evidence of its effectiveness at reducing FI among an at-risk minority population, in whom limited evidence exists, and during the ARRA SNAP expansion period. To the best of our knowledge, only two other studies have investigated food security changes in response to ARRA SNAP expansion, using data from the Current Population Survey ${ }^{(2,6)}$. Similar to our findings, one of these studies showed that participation in SNAP increased and FI decreased for low-income households from pre- to postARRA SNAP expansion ${ }^{(2)}$. Similar shifts were also observed for mixed-status Mexican households but not for Mexican immigrant households who are likely not eligible due to documentation status ${ }^{(6)}$, underscoring the importance of FI research focused on vulnerable groups.

For sustained and new participants in our sample, total carbohydrate intake was higher post-ARRA, compared with discontinued participants. Waehrer et al. found no differences for total carbohydrates post-ARRA SNAP expansion among SNAP-eligible NHANES households ${ }^{(5)}$. Higher carbohydrate intake for sustained participants was likely from higher-quality food sources, as their dietary fibre intake was also higher post-ARRA compared with discontinued participants. Notably, the higher carbohydrate and fibre intakes may be due to higher intake of a combination of fruits, vegetables and whole grains, as these tended to be higher among sustained and new participants 
Table 2 Mean nutrient intakes and AHEI-2010 scores, with their SE, post-ARRA SNAP expansion by SNAP participation status, adjusted for pre-ARRA nutrient intake, sociodemographic, sociocultural and health factors, in the Boston Puerto Rican Health Study ( $n$ 402)†

\begin{tabular}{|c|c|c|c|c|c|c|}
\hline & \multicolumn{2}{|c|}{ Sustained ( $n$ 249) } & \multicolumn{2}{|c|}{ New $(n 95)$} & \multicolumn{2}{|c|}{ Discontinued ( $n$ 58) } \\
\hline & Mean & SE & Mean & SE & Mean & SE \\
\hline Total energy $(\mathrm{kJ} / \mathrm{d})$ & 8042 & 234 & 8125 & 314 & 7958 & 402 \\
\hline Total energy $(\mathrm{kcal} / \mathrm{d})$ & 1922 & 56 & 1942 & 75 & 1902 & 96 \\
\hline Total fat $(\mathrm{g} / \mathrm{d})$ & 54 & 0.6 & 54 & 0.8 & 56 & $1 \cdot 1$ \\
\hline $\mathrm{SFA}(\mathrm{g} / \mathrm{d})$ & $16 \cdot 2$ & 0.3 & $16 \cdot 0$ & 0.4 & $16 \cdot 9$ & 0.5 \\
\hline PUFA (g/d) & $13 \cdot 7$ & 0.2 & $13 \cdot 9$ & 0.3 & $14 \cdot 0$ & 0.4 \\
\hline MUFA $(\mathrm{g} / \mathrm{d})$ & $19 \cdot 3$ & 0.2 & 19.5 & 0.3 & $20 \cdot 2$ & 0.4 \\
\hline$n-3$ Fatty acids $(\mathrm{g} / \mathrm{d})$ & 1.3 & 0.03 & 1.2 & 0.03 & 1.3 & 0.04 \\
\hline Trans-fatty acids $(\mathrm{g} / \mathrm{d})$ & 1.9 & 0.1 & $1 . \overline{9}$ & 0.1 & $2 \cdot 0$ & 0.1 \\
\hline Protein $(\mathrm{g} / \mathrm{d})$ & $68 \cdot 8$ & 0.9 & 67.5 & $1 \cdot 2$ & $69 \cdot 7$ & 1.5 \\
\hline Animal protein $(\mathrm{g} / \mathrm{d})$ & $46 \cdot 0$ & 0.9 & $44 \cdot 2$ & $1 \cdot 3$ & $47 \cdot 8$ & 1.6 \\
\hline Plant protein $(\mathrm{g} / \mathrm{d})$ & $22 \cdot 7$ & 0.3 & 23.2 & 0.5 & 21.8 & 0.6 \\
\hline Total carbohydrates $(\mathrm{g} / \mathrm{d})$ & $215^{\star *}$ & 1.9 & $216^{*}$ & 2.6 & 204 & 3.4 \\
\hline Dietary fibre $(\mathrm{g} / \mathrm{d})$ & $19 \cdot 0^{*}$ & 0.4 & 18.9 & 0.5 & 17.5 & 0.6 \\
\hline Added sugars $(\mathrm{g} / \mathrm{d})$ & $43 \cdot 1$ & $2 \cdot 0$ & $42 \cdot 4$ & $2 \cdot 6$ & $37 \cdot 7$ & 3.4 \\
\hline Starches $(g / d)$ & 95.8 & 1.4 & $99 \cdot 2$ & 1.9 & $95 \cdot 1$ & $2 \cdot 4$ \\
\hline Alcohol $(\mathrm{g} / \mathrm{d})$ & $4 \cdot 5^{\star \star}$ & 0.8 & $5 \cdot 1^{*}$ & $1 \cdot 1$ & 9.7 & 1.5 \\
\hline \multicolumn{7}{|l|}{ Micronutrients } \\
\hline Vitamin A $(\mu \mathrm{g} / \mathrm{d})$ & 8925 & 437 & 7436 & 591 & 8925 & 754 \\
\hline Vitamin D $(\mu \mathrm{g} / \mathrm{d})$ & 6.3 & 0.4 & $6 \cdot 1$ & 0.5 & 6.6 & 0.7 \\
\hline Vitamin $B_{12}(\mu \mathrm{g} / \mathrm{d})$ & $17 \cdot 0$ & 4.5 & 17.5 & $6 \cdot 1$ & $16 \cdot 6$ & $7 \cdot 8$ \\
\hline Vitamin $B_{6}(\mu / \mathrm{dg})$ & $2 \cdot 3$ & 0.5 & 1.9 & 0.6 & 3.2 & 0.8 \\
\hline Folate $(\mu \mathrm{g} / \mathrm{d})$ & 423 & 10 & 426 & 14 & 409 & 18 \\
\hline $\mathrm{Ca}(\mathrm{mg} / \mathrm{d})$ & 810 & 29 & 764 & 40 & 841 & 51 \\
\hline $\mathrm{Mg}(\mathrm{mg} / \mathrm{d})$ & 273 & 4.5 & 269 & $6 \cdot 0$ & 262 & $7 \cdot 7$ \\
\hline $\mathrm{Na}(\mathrm{mg} / \mathrm{d})$ & 3267 & 50 & 3275 & 68 & 3243 & 86 \\
\hline $\mathrm{K}(\mathrm{mg} / \mathrm{d})$ & 2682 & 38 & 2613 & 51 & 2573 & 65 \\
\hline $\mathrm{Fe}(\mathrm{mg} / \mathrm{d})$ & $16 \cdot 0$ & 0.8 & 18.4 & $1 \cdot 1$ & $17 \cdot 8$ & 1.5 \\
\hline AHEI-2010 score (and component scores)‡ & $52 \cdot 0$ & 0.5 & $51 \cdot 8$ & 0.7 & $50 \cdot 3$ & 0.9 \\
\hline Vegetables & 4.6 & 0.2 & 4.3 & 0.2 & $4 \cdot 2$ & 0.3 \\
\hline Fruit & $2 \cdot 3$ & $0 \cdot 1$ & $2 \cdot 2$ & 0.1 & 1.9 & 0.2 \\
\hline Whole grains & $1 \cdot 3$ & $0 \cdot 1$ & 1.4 & 0.1 & $1 \cdot 2$ & 0.1 \\
\hline Sugar-sweetened beverages and fruit juice & $3 \cdot 1$ & 0.2 & $3 \cdot 2$ & 0.3 & 2.9 & 0.4 \\
\hline Nuts and legumes & 3.9 & 0.2 & $4 \cdot 1$ & 0.2 & $4 \cdot 0$ & 0.3 \\
\hline Red/processed meat & 4.9 & 0.2 & $5 \cdot 1$ & 0.2 & $4 \cdot 7$ & 0.3 \\
\hline Trans-fatty acids & 8.4 & 0.1 & 8.4 & 0.1 & 8.3 & 0.1 \\
\hline$n-3$ Fatty acids & $5 \cdot 4$ & 0.1 & $5 \cdot 3$ & 0.1 & $5 \cdot 7$ & 0.2 \\
\hline PUFA & $6 \cdot 7$ & 0.1 & $6 \cdot 8$ & 0.2 & $6 \cdot 8$ & 0.2 \\
\hline $\mathrm{Na}$ & $7 \cdot 7$ & 0.1 & $7 \cdot 8$ & 0.1 & $7 \cdot 7$ & 0.1 \\
\hline Alcohol & $3 \cdot 6^{* *}$ & 0.1 & 3.3 & 0.2 & 2.9 & 0.2 \\
\hline
\end{tabular}

AHEI-2010, Alternate Healthy Eating Index-2010; ARRA, 2009 American Recovery and Reinvestment Act; SNAP, Supplemental Nutrition Assistance Program. ${ }^{\star} P<0.05,{ }^{\star \star} P<0.01$ compared with discontinued participants.

†Linear repeated-measures models (with Tukey adjustment for multiple comparisons) and general estimating equations adjusted for pre-ARRA nutrient intake, age, sex, income-to-poverty ratio, educational attainment, psychological acculturation, health insurance, depressive symptoms and BMI.

$\ddagger$ The AHEl-2010 assessed dietary quality using eleven dietary components. Higher scores indicate higher diet quality (total score range from 0 to 110 ). Each individual food or nutrient component is scored 0 to 10 (higher value indicating healthier intake).

post-ARRA compared with discontinued participants, although the differences did not reach statistical significance. These findings again contrast with those from low-income, SNAP-eligible households in NHANES, in that the post-ARRA SNAP expansion period was not associated with higher fibre intake ${ }^{(5)}$ although the comparison with our findings is limited because the report on NHANES data did not consider SNAP participation, only eligibility. Mainland US Puerto Ricans have lower intake of fibre than other Hispanic/Latino heritages in the USA ${ }^{(24)}$. Thus, our findings suggest that increasing monthly benefits for Puerto Rican adults participating in SNAP may be an important strategy for improving fibre intake. Future programmatic research should investigate which fibre-rich foods are acceptable and feasible to increase among mainland
US Puerto Ricans participating in SNAP, and how those foods are incorporated into daily life, although previous research in this cohort suggests that sources include beans and legumes, high-fibre cereals, oatmeal and whole-wheat bread $^{(25)}$.

We did not document significant post-ARRA differences in mean diet quality score or component scores by SNAP participant group, except for alcohol intake. ARRA appeared to negatively impact mean diet quality score of low-income, SNAP-eligible households in NHANES, with a stronger effect among households at the lowest level (25th percentile) of diet quality pre-ARRA ${ }^{(5)}$. Likewise, SNAP-eligible NHANES households with low intakes (25th percentile) of fruits and vegetables pre-ARRA had a decrease in fruit and vegetable intakes post-ARRA SNAP 
expansion ${ }^{(5)}$. Although the differences were not statistically significant, intakes of fruits and vegetables tended to be higher for sustained and new SNAP participants in our sample. Even modest, non-significant higher intakes of fruits and vegetables among US Hispanic/Latino SNAP participants of Puerto Rican heritage would be important, as they have lower fruit and vegetable intakes compared with other Hispanic/Latino heritages ${ }^{(10)}$ and high rates of SNAP participation ${ }^{(8)}$. Our findings underscore the need for further research on the potential nutritional benefit of ARRA SNAP expansions among diverse SNAP participants and for more targeted approaches to improving nutritional intake among low-income populations.

Our findings of lower alcohol intake among sustained and new participants compared with discontinued participates are novel. SNAP-eligible households in NHANES did not differ post-ARRA for intake of a food category combining saturated fats, alcohol and added sugars ${ }^{(5)}$. We also did not find differences post-ARRA SNAP expansion for intakes of saturated fat, added sugars, and sugar-sweetened beverages or fruit juices, but our observed lower alcohol intake demonstrates not only the importance of disaggregating certain foods and nutrients but also the broader implications of SNAP participation. SNAP restricts alcohol purchases, possibly influencing the lower intake of alcohol among sustained and new participants compared with discontinued participants, although participants with alcohol use disorders may have been more likely to discontinue their SNAP participation. A recent study reported an inverse relationship between alcohol expenditure and household food purchasing across income quintiles ${ }^{(26)}$, suggesting that individuals in our sample with discontinued SNAP participation and higher alcohol intake may have been at a higher dietary and FI risk, which may help explain the lower intakes of total carbohydrates and fibre we observed. Alcohol consumption among low-income adults in 2005-2008 NHANES did not differ by SNAP participation ${ }^{(27)}$ but most of these NHANES cycles were collected before the great recession and may not provide an adequate comparison with our findings, as alcohol use and problems increased as a function of economic losses during the recession ${ }^{(28)}$. Furthermore, mainland US Puerto Ricans report a higher average number of drinks per week and a greater proportion of binge drinking compared with other Hispanic/Latino heritages ${ }^{(29)}$ and have higher incidence of alcohol use disorders compared with other Hispanic/ Latino heritages and non-Hispanic Whites ${ }^{(30)}$. Lower alcohol intake among SNAP participants in our sample of Puerto Rican adults underscores the need for more research in highly vulnerable SNAP-eligible populations and more evaluations of the potential impacts of SNAP participation beyond improving food security.

The post-ARRA SNAP expansion improvements for some nutrients in our cohort contribute to a limited evidence base on dietary intake among Hispanic/Latino adults participating in SNAP, most of which is cross-sectional and shows poorer dietary intake compared with eligible non-participants ${ }^{(31,32)}$. In a crosssectional sample of low-income, Hispanic/Latino young and middle-aged women in Texas, SNAP participants consumed more total sugars and less whole grains compared with non-participants ${ }^{(32)}$. However, the non-participant category may have contained both SNAP-eligible and SNAP-ineligible women and, thus, the comparison with SNAP participants should be interpreted carefully. Considering most Hispanics/Latinos residing in Texas are of Mexican heritage, the contrasting findings reiterate the importance of potential Hispanic/Latino heritagespecific differences in dietary intake among SNAP participants. More than half ( $52 \%$ ) of SNAP-eligible households in NHANES during the ARRA SNAP expansion were nonHispanic Black, Hispanic or of other races ${ }^{(5)}$, but no tests of racial or ethnic differences were reported. NHANES data from 2003-2010 found higher diet quality scores among non-Hispanic White adults participating in SNAP compared with eligible, non-participant non-Hispanic White adults, but similar differences were not observed between non-Hispanic Black or Hispanic SNAP participants $v$. their eligible non-participant counterparts ${ }^{(33)}$, suggesting that non-Hispanic White SNAP participants benefited more than non-Hispanic Black or Hispanic SNAP participants. The authors acknowledged that one potential explanation of the differences may be disparities in healthy food access. For Hispanics/Latinos, healthy food access and its impact on dietary intake may be distinctly tied to higher-density ethnic composition of the neighbourhood ${ }^{(34)}$, food store appearance and the quality of available fresh produce ${ }^{(35)}$. Although evidence among adult SNAP participants suggests overall lower diet quality scores compared with non-participants ${ }^{(4)}$, additional research is needed among ethnic minority populations participating in SNAP to understand potential differences in dietary intake and food access that can be better targeted in SNAP programming.

The documented decrease in FI and healthier nutrient intake for SNAP participants post-ARRA SNAP expansion in this sample of Puerto Ricans has potential positive downstream health implications. Previous research in this cohort has shown that food-insecure participants with diabetes had significantly lower diet quality scores compared with food-secure participants with diabetes, and this lower diet quality was associated with poorer glycaemic control $^{(36)}$. Likewise, AHEI-2010 was inversely associated with 2-year cardiometabolic risk factors in this cohort $^{(25)}$, further demonstrating the important role of healthier diets in preventing and reducing cardiometabolic risk in a low-income population. Low-income households consistently make trade-offs between food security, housing bills and medication ${ }^{(37,38)}$. SNAP continuity, during volatile times, and expanded eligibility and benefits may more effectively provide the necessary safety net that vulnerable households need to access 
sufficient, healthier foods without employing detrimental trade-offs. Thus, an adequately funded and executed food assistance programme, such as SNAP, has the potential to positively influence the health of individuals in low-income households through greater adherence to necessary medication ${ }^{(39)}$, more resources allocated for healthy food that can protect against cardiometabolic diseases, attenuation of stress related to $\mathrm{FI}^{(8)}$ and reduction of unhealthy behaviours (e.g. excessive alcohol intake) often the consequence of stress and poverty $^{(28,30)}$. As a result, SNAP expansions have the potential to contribute healthier individuals to the US workforce ${ }^{(40)}$ and to reduce US health disparities and the associated \$US 229 billion in direct medical care expenditures $^{(41)}$.

Our study had several limitations. First, our sample size was small, preventing us from having an ideal comparison group, either eligible non-participants or ineligible nonparticipants with incomes between 150 and $250 \%$ of the federal poverty line. New and discontinued participant categories were also small and may be a reason we did not detect many changes in nutrient intakes and AHEI-2010 scores by SNAP participation status, although testing differences by participation status rather than by eligibility provided unique insights. Additionally, preARRA data on FI were from the baseline visit, collected between 2004 and 2007, and may have resulted in some participants being misclassified immediately preceding ARRA initiation.

Our study had several strengths. First, the study used a valid and reliable assessment of FI, the ten-item adult food security survey module which is used to track national FI prevalence ${ }^{(13)}$, and a valid assessment of dietary intake for Puerto Ricans ${ }^{(42)}$. Likewise, we were able to adjust our models for a number of important factors correlated with SNAP participation and dietary intake, and influential to low-income Puerto Ricans in this cohort. The longitudinal design provided the opportunity to assess postARRA values while controlling for pre-ARRA values, rather than assessing values at two time points using different cross-sectional samples, like NHANES. Additionally, because of the large proportion of individuals in this cohort participating in SNAP, we were able to test differences by sustained, new or discontinued participation status, rather than solely eligibility.

\section{Conclusions}

Our findings demonstrate the importance of SNAP continuity and expanded eligibility and benefits, especially during an economic downturn, in protecting individuals and households from FI, as well as positively shaping dietary intake. Future research should add to our findings by addressing adequacy of SNAP benefits ${ }^{(43)}$, investigating the added impact of participation in SNAP-education programming, focusing more on vulnerable and minority populations, and reducing barriers to SNAP participation.

\section{Acknowledgements}

Acknowledgements: The authors thank Dr Sara Bleich for her input on the methodological approach.Financial support: The BPRHS was supported by the National Institutes of Health (NIH), National Institute on Aging (grant number P01-AG023394); and by the US Department of Agriculture, Agriculture Research Institute (grant number 58-1950-7-707). A.C.M. received support for this study from an NIH Ruth L. Kirschstein Institutional Training Grant Postdoctoral Fellowship (grant number 5 T32 DK 770323). J.M. received support for this study from an NIHNational Heart, Lung, and Blood Institute Mentored Career Development Award to Promote Faculty Diversity (grant number K01-HL120951). The NIH and the USDA had no role in the design, writing or analysis of this article. Conflict of interest: None. Authorship: A.C.M. developed the study questions and design, analysed and interpreted the data, and wrote the manuscript. J.M. assisted with developing the study questions and design, interpreting the data, and critically revising the manuscript. L.M.F. and K.L.T. contributed to the study questions and design and to revisions of the manuscript. All authors read and approved the final manuscript. Ethics of human subject participation: This study was conducted according to the guidelines laid down in the Declaration of Helsinki and all procedures involving human subjects were approved by the Institutional Review Boards at Tufts Medical Center and at Northeastern University. Written informed consent was obtained from all subjects.

\section{Supplementary material}

To view supplementary material for this article, please visit https://doi.org/10.1017/S1368980019002209

\section{References}

1. Kumcu A \& Kaufman P (2011) Food spending adjustments during recessionary times. Amber Waves 9, 10-17.

2. Nord M \& Prell M (2011) Food Security Improved Following the 2009 ARRA Increase in SNAP Benefits. Economic Research Report no. ERR-116. Washington, DC: US Department of Agriculture, Economic Research Service; available at https://www.ers.usda.gov/publications/pubdetails/?pubid=44839 (accessed March 2018).

3. Alaimo K (2005) Food insecurity in the United States: an overview. Top Clin Nutr 20, 281-298.

4. Andreyeva T, Tripp AS \& Schwartz MB (2015) Dietary quality of Americans by supplemental nutrition assistance program participation status: a systematic review. Am J Prev Med $\mathbf{4 9}$, 594-604. 
5. Waehrer G, Deb P \& Decker SL (2015) Did the 2009 American Recovery and Reinvestment Act affect dietary intake of lowincome individuals? Econ Hum Biol 19, 170-183.

6. Kaushal N, Waldfogel J \& Wight V (2014) Food insecurity and SNAP participation in Mexican immigrant families: the impact of the outreach initiative. B EJ Econom Anal Policy 14, 203-240.

7. Rabbitt MP, Smith MD \& Coleman-Jensen A (2016) Food Security Among Hispanic Adults in the United States, 2011-2014. Economic Information Bulletin no. EIB-153. Washington, DC: US Department of Agriculture, Economic Research Service; available at https://www.ers.usda. gov/webdocs/publications/44080/59326_eib-153.pdf?v=0 (accessed May 2016).

8. McClain AC, Xiao RS, Gao X et al. (2018) Food insecurity and odds of high allostatic load in Puerto Rican adults: the role of participation in the supplemental nutrition assistance program during 5 years of follow-up. Psychosom Med 80, 733-741.

9. Bermudez OI, Falcon LM \& Tucker KL (2000) Intake and food sources of macronutrients among older Hispanic adults: association with ethnicity, acculturation, and length of residence in the United States. J Am Diet Assoc 100, 665-673.

10. Mattei J, Sotres-Alvarez D, Daviglus ML et al. (2016) Diet quality and its association with cardiometabolic risk factors vary by Hispanic and Latino ethnic background in the Hispanic community health study/study of Latinos. J Nutr 146, 2035-2044.

11. Daviglus ML, Talavera GA, Aviles-Santa ML et al. (2012) Prevalence of major cardiovascular risk factors and cardiovascular diseases among Hispanic/Latino individuals of diverse backgrounds in the United States. JAMA 308, 1775-1784.

12. Tucker KL, Mattei J, Noel SE et al. (2010) The Boston Puerto Rican Health Study, a longitudinal cohort study on health disparities in Puerto Rican adults: challenges and opportunities. BMC Public Health 10, 107.

13. Hamilton WL, Cook JT, Thompson WW et al. (1997) Household Food Security in the United States in 1995: Technical Report of the Food Security Measurement Project. Alexandria, VA: US Department of Agriculture, Food and Consumer Service; available at https://www.fns.usda.gov/ household-food-security-united-states-1995-summary-reportfood-security-measurement-project (accessed May 2018).

14. US Department of Agriculture, Economic Research Service (2012) US Adult Food Security Survey Module: Three-Stage Design, with Screeners. https://www.ers.usda.gov/media/ 8279/ad2012.pdf (accessed January 2017).

15. Hanson KL \& Connor LM (2014) Food insecurity and dietary quality in US adults and children: a systematic review. Am J Clin Nutr 100, 684-692.

16. Tucker KL, Bianchi LA, Maras J et al. (1998) Adaptation of a food frequency questionnaire to assess diets of Puerto Rican and non-Hispanic adults. Am J Epidemiol 148, 507-518.

17. Rhee JJ, Cho E \& Willett WC (2014) Energy-adjustment of nutrient intakes is preferable to adjustment using body weight and physical activity in epidemiological analyses. Public Health Nutr 17, 1054-1060.

18. Chiuve SE, Fung TT, Rimm EB et al. (2012) Alternative dietary indices both strongly predict risk of chronic disease. J Nutr 142, 1009-1018.

19. US Census Bureau (2016) How the Census Bureau Measures Poverty. https://www.census.gov/topics/incomepoverty/poverty/guidance/poverty-measures.html (accessed December 2016).

20. Tropp LR, Erkut S, Coll CG et al. (1999) Psychological acculturation: development of a new measure for Puerto Ricans on the US mainland. Educ Psychol Meas 59, 351-367.
21. Radloff LS (1977) The CES-D Scale: a self-report depression scale for research in the general population. Appl Psychol Meas 1, 385-401.

22. Tucker KL, Bermudez OI \& Castaneda C (2000) Type 2 diabetes is prevalent and poorly controlled among Hispanic elders of Caribbean origin. Am J Public Health 90, 1288-1293.

23. Mabli J \& Ohls J (2015) Supplemental Nutrition Assistance Program participation is associated with an increase in household food security in a national evaluation. $J$ Nutr 145, 344-351.

24. Siega-Riz AM, Sotres-Alvarez D, Ayala GX et al. (2014) Foodgroup and nutrient-density intakes by Hispanic and Latino backgrounds in the Hispanic Community Health Study/ Study of Latinos. Am J Clin Nutr 99, 1487-1498.

25. Mattei J, Sotos-Prieto M, Bigornia SJ et al. (2017) The Mediterranean diet score is more strongly associated with favorable cardiometabolic risk factors over 2 years than other diet quality indexes in Puerto Rican adults. J Nutr 147, 661-669.

26. Gell L \& Meier P (2012) The nature and strength of the relationship between expenditure on alcohol and food: an analysis of adult-only households in the UK. Drug Alcohol Rev 31, 422-430.

27. Todd JE \& Ver Ploeg M (2014) Caloric beverage intake among adult Supplemental Nutrition Assistance Program participants. Am J Public Health 104, e80-e85.

28. Mulia N, Zemore SE, Murphy R et al. (2014) Economic loss and alcohol consumption and problems during the 2008-9 US Recession. Alcohol Clin Exp Res 38, 1026-1034.

29. Ramisetty-Mikler S, Caetano R \& Rodriguez LA (2010) The Hispanic Americans Baseline Alcohol Survey (HABLAS): alcohol consumption and sociodemographic predictors across Hispanic national groups. J Subst Use 15, 402-416.

30. Ríos-Bedoya CF \& Freile-Salinas D (2014) Incidence of alcohol use disorders among Hispanic subgroups in the USA. Alcohol Alcohol 49, 549-556.

31. Nguyen BT, Shuval K, Njike VY et al. (2014) The Supplemental Nutrition Assistance Program and dietary quality among US adults: findings from a nationally representative survey. Mayo Clin Proc 89, 1211-1219.

32. Hilmers A, Chen T-A, Dave JM et al. (2014) Supplemental Nutrition Assistance Program participation did not help low income Hispanic women in Texas meet the dietary guidelines. Prev Med 62, 44-48.

33. Nguyen BT, Shuval K, Bertmann F et al. (2015) The Supplemental Nutrition Assistance Program, food insecurity, dietary quality, and obesity among US adults. Am J Public Health 105, 1453-1459.

34. Park Y, Neckerman K, Quinn J et al. (2011) Neighbourhood immigrant acculturation and diet among Hispanic female residents of New York City. Public Health Nutr 14, 1593-1600.

35. Sharif MZ, Albert SL, Chan-Golston AM et al. (2017) Community residents' beliefs about neighborhood corner stores in 2 Latino communities: implications for interventions to improve the food environment. J Hunger Environ Nutr 12, 342-351.

36. Berkowitz SA, Gao X \& Tucker KL (2014) Food-insecure dietary patterns are associated with poor longitudinal glycemic control in diabetes: results from the Boston Puerto Rican Health Study. Diabetes Care 37, 2587-2592.

37. Olson CM (2005) Food insecurity in women: a recipe for unhealthy trade-offs. Top Clin Nutr 20, 321-328.

38. Nord M \& Kantor LS (2006) Seasonal variation in food insecurity is associated with heating and cooling costs among low-income elderly Americans. J Nutr 136, 2939-2944.

39. Pooler JA \& Srinivasan M (2019) Association between Supplemental Nutrition Assistance Program participation and cost-related medication nonadherence among older adults with diabetes. JAMA Intern Med 179, 63-70.

40. Sullivan PW, Ghushchyan V, Wyatt HR et al. (2007) Productivity costs associated with cardiometabolic risk factor clusters in the United States. Value Health 10, 443-450. 
41. LaVeist TA, Gaskin D \& Richard P (2011) Estimating the economic burden of racial health inequalities in the United States. Int J Health Serv 41, 231-238.

42. Tucker KL, Bianchi LA, Maras J et al. (1998) Adaptation of a food frequency questionnaire to assess diets of Puerto Rican and non-Hispanic adults. Am J Epidemiol 148, 507-518.
43. Institute of Medicine \& National Research Council (2013) Supplemental Nutrition Assistance Program: Examining the Evidence to Define Benefit Adequacy. Washington, DC: The National Academies Press; available at https://www.nap.edu/ catalog/13485/supplemental-nutrition-assistance-programexamining-the-evidence-to-define-benefit (accessed March 2017). 